\title{
A comparison of Drug Eluting Stents with different coatings in rabbit iliac artery model
}

Background: New generation of Drug Eluting Stents significantly reduce restenosis as compared to Bare-metal stents. At the same time, a number of clinical studies demonstrated dependence between strut endothelialization rate and rate of late stent thrombosis. Modern stent developers and manufacturers have limited number of materials for stent coating. Theoretically it could be assumed that similar drug polymer formulation may yield similar biological effect and clinical results and it is even possible to extrapolate clinical data from one stent model to the others (with same coating formulation).

Methods and findings: We evaluated the biological response and endothelization rate of different DES with various drug-polymer formulations after implantation in rabbit iliac artery. Coating surface morphology of each stent was assessed by scanning electron microscopy (SEM) at 14 and 28-day of implantation.

Conclusions: It was found that DES with similar drug-polymer formulation but different morphology demonstrated different results after implantation. At the same time, DES with different coating formulation but similar morphology demonstrated similar biological effect. Thus, our study confirms that apart from coating formulation, coating morphology and topology are critical for stent biocompatibility and endothelization rate. Our future work will be devoted to detailed in vivo study of influence of the rate of polymer coating degradation on vascular healing.

Keywords: Drug-eluting stents - Rabbit iliac model - Scanning electron microscopy - Polymer coating defects - Stent endothelization

\section{Introduction}

The majority of clinical trials conducted in recent years demonstrated advantages of second-generation coronary drug-eluting stents (DES) over bare-metal stents (BMS) in reducing restenosis rate by suppressing neo-intimal growth in stented segment of the vessel in post-implantation period [1]. One may expect that stents with similar coating composition, such as polylactide-based copolymer and identical drug (Sirolimus) would demonstrate similar physicochemical properties and biological response as well, but there is a lack of experimental evidence in this aspect. For an instance, stent coating defects could lead to abnormal vascular responses and delay the endothelialization. This was evidenced by a series of studies conducted by Bazalus et al. [2].

Recent study of different DES with polymer coating demonstrated [3] application of similar polymers and similar drugs for coating may vary the surface morphology (smoothness, number and types of defects and irregularities) in bench testing experiments.

The purpose of this study is to evaluate the biological response and endothelialization and coating integrity after implantation of different DES. Two drug-eluting stents with
Dmitry $\vee$ Kapustin ${ }^{1 *}$, Natalia Yu Orlinskaya ${ }^{2}$ and Kseniya $V$ Kulakova ${ }^{2}$

'Shemyakin-Ovchinnikov Institute of Bioorganic Chemistry of the Russian Academy of Sciences, Moscow, Russia 2Privolzhsky Federal Research Medical Centre of the Ministry of Health of the Russian Federation, Federal State Budgetary Institution, Nizhny Novgorod, Russia

*Author for correspondence:

Tel.: +79162776094

kapustin@ibch.ru

Submitted: 02 November 2017

Accepted: 29 December 2017

Published online: 05 January 2018 
similar biodegradable polylactide-based sirolimuseluting coating-Calipso (Angiolain, Russia) and Biomime (Meril, India) were investigated and compared with Resolute Integrity (Medtronic, USA)-DES stent with durable polymer-based zotarolimus-eluting coating with excellent clinical outcomes [4].

\section{Materials and Methods}

\section{Objects of research}

The details of the stents applied for this research work is described in the following section:

1. Stent \#1: Endovascular metal stent with polymeric drug-eluting coating-Calipso (Angioline, Russia). The Stent size was: $2.50 \times 13$ $\mathrm{mm}$. Coating composition used for the stent: biodegradable copolymer of glycolic and lactic acids. Drug substance applied: Sirolimus, labeled amount-150 $\mu \mathrm{g} / \mathrm{cm}^{2}\left(1.25 \mu \mathrm{g} / \mathrm{mm}^{2}\right)$. Full drug release details were unknown.

2. Stent \#2: Endovascular metal stent with polymeric drug-eluting coating-Biomime (Meril, India). Stent size was: $2.50 \times 13 \mathrm{~mm}$. Coating composition used: polymer-BioPoly ${ }^{\mathrm{TM}}$, biodegradable copolymer of glycolic and lactic acids. Drug substance used: Sirolimus, labeled amount-1.25 $\mu \mathrm{g} / \mathrm{mm}^{2}$. Full drug release occurred in 30 days.

3. Stent \#3 (Control): Endovascular metal stent with durable polymeric drug-eluting coating - Resolute Integrity, (Medtronic, USA). Stent size was: $2.50 \times 12 \mathrm{~mm}$. Drug coating applied: durable polymer BioLinx ${ }^{\mathrm{TM}}$-blend of the components C10 and C19, and PVP (polyvinyl pyrrolidone). Drug substance used: Zotarolimus, labeled amount-1.6 $\mu \mathrm{g} / \mathrm{mm}^{2}$. Complete drug release occurred within 180 days.

\section{Implantation}

The study protocol was approved by the Local Bioethics Committee of the Privolzhsky Federal Research Medical Center of Ministry of Health of the Russian Federation (Nizhniy Novgorod).

A total of 40 stents were implanted in 20 adult male rabbits without atherosclerotic vascular injury. Male Chinchilla rabbits were anesthetized with Zoletil and Xylavet. Endovascular procedure and procedure of euthanasia (after 14 days, $\mathrm{n}=10$ and 28 days, $\mathrm{n}=10$ ) were conducted as described by Lee et al. [5]. Drug eluting stents (DES), i.e., biodegradable polylactide- based sirolimus-eluting stents (Stent \#1-Calypso, Angioline, Russia, and Stent \#2-Meril, Biomime, India), and the durable polymer-based zotarolimuseluting stent was used as a control stent (Stent \#3Resolute Integrity, Medtronic, USA). Stents were implanted in the left and right iliac arteries (two stents in each rabbit, one with the biodegradable coating and one with durable coating). Stents were deployed according to the recommendations of manufactures to their nominal diameters to achieve stent-to-artery ratio of 1.1:1. Geparin and antibiotic (Cefazolin-AKOS) were administered after implantation.

The animals were sacrificed at the chosen study time points after anesthetization. The iliac artery segments containing the stent were dissected, removed, cleaned from periadventitial fragments and fixed in 10\% neutral buffered formaline.

\section{Scanning electron microscopy (SEM)}

SEM (Scanning Electron Microscope) characterization was carried out on a JEOL JSMIT300LV (JEOL Japanese Electron Optics Laboratory) in high vacuum mode and at low probe current values $(<0.1 \mathrm{nA})$ to reduce the impact of an electron beam on the investigated samples. X-ray microanalysis of individual sections was performed with SDD (Silicon Drift Detector) XMax N-20 and AZtec analysis software (Oxford Instruments, UK).

\section{Measurement of endothelialized strut area:}

Measurement of the endothelialized strut area was conducted at $14^{\text {th }}$ day and $28^{\text {th }}$ day. The percentage of uncovered struts area was calculated from lowmagnification SEM images of vessels at $14^{\text {th }}$ day and $28^{\text {th }}$ day.

SEM images were traced with Adobe Photoshop software (Adobe, USA). The total stented area, as well as area of the uncovered parts of the stent struts was measured as described by Lee et al. [5]. The percentage of uncovered struts was calculated as:

Percentage $(\%)$ uncovered struts $=($ area of uncovered struts/total stented area) $\times 100 \%$.

\section{Statistical analysis}

All values were presented as Mean \pm SD where $n=5$. As the dataset obtained was containing non-normally distributed data, we used Mann-Whitney U-test for analyzing difference between samples. $\mathrm{P}<0.05$ was considered as statistically significant. 


\section{Results}

SEM imaging provided the general view across the lumen of the stented vessel segments. The fixed segments of inner wall of the vessel with the stent fragment and the areas of the struts uncovered by the newly formed endothelium was also analysed through this visualization.

Segments of 40 stented arteries, $(20$ segments from 10 animals after 14 days with Stent \#1 and Stent \#2$\mathrm{n}=5$ for each type, and Control-n=10; 20 segments from 10 animals after 28 days with Stent \#1 and Stent $\# 2-n=5$ for each type, and Control-n=10) were cut longitudinally to expose the luminal surface. These were placed in chamber of microscope and images were taken at different magnifications $(\times 10-15, \times 50, \times 150-200)$. For more accurate estimation of the implanted stents we used two types of detectors, backward-reflected electron detector (BED) and secondary electron detector (SED) for each sample.

The images obtained with BED allowed getting information on the compositional contrast in the investigated regions of the stents which were not covered by the newly formed endothelium. SED was used to obtain the information about the sample topographic characteristics. For individual sections, the low-vacuum secondary electron (LVSED) signal was used to reduce the effects of electrostatic charging of the sample surface, which adversely affect the image.

In presented in vivo study thrombus formation was not observed in any stent, however, different stents demonstrated different proportion of endothelium covered/uncovered area.

\section{Stents evaluation at $\mathbf{1 4}$ days}

The analysis of SEM images is presented in Figure 1. Thus, at 14 day none of the stents demonstrated the full covering of endothelium, however, as provided in Figures $1 \mathrm{~B}$ and $1 \mathrm{C}$, the Stent $\# 1$ has the highest value of uncovered areas. The lowest value of uncovered area was for Control $(0.9 \% \pm 0.6 \%)$ and the difference between Stent \#1 and Stent \#2 was significant $(2.75 \% \pm 2.38$ vs. $1.37 \% \pm 1.14 \%, \mathrm{P}=0.01)$. The obtained results are consistent with the results published earlier for atherosclerotic rabbit iliac artery model [7].
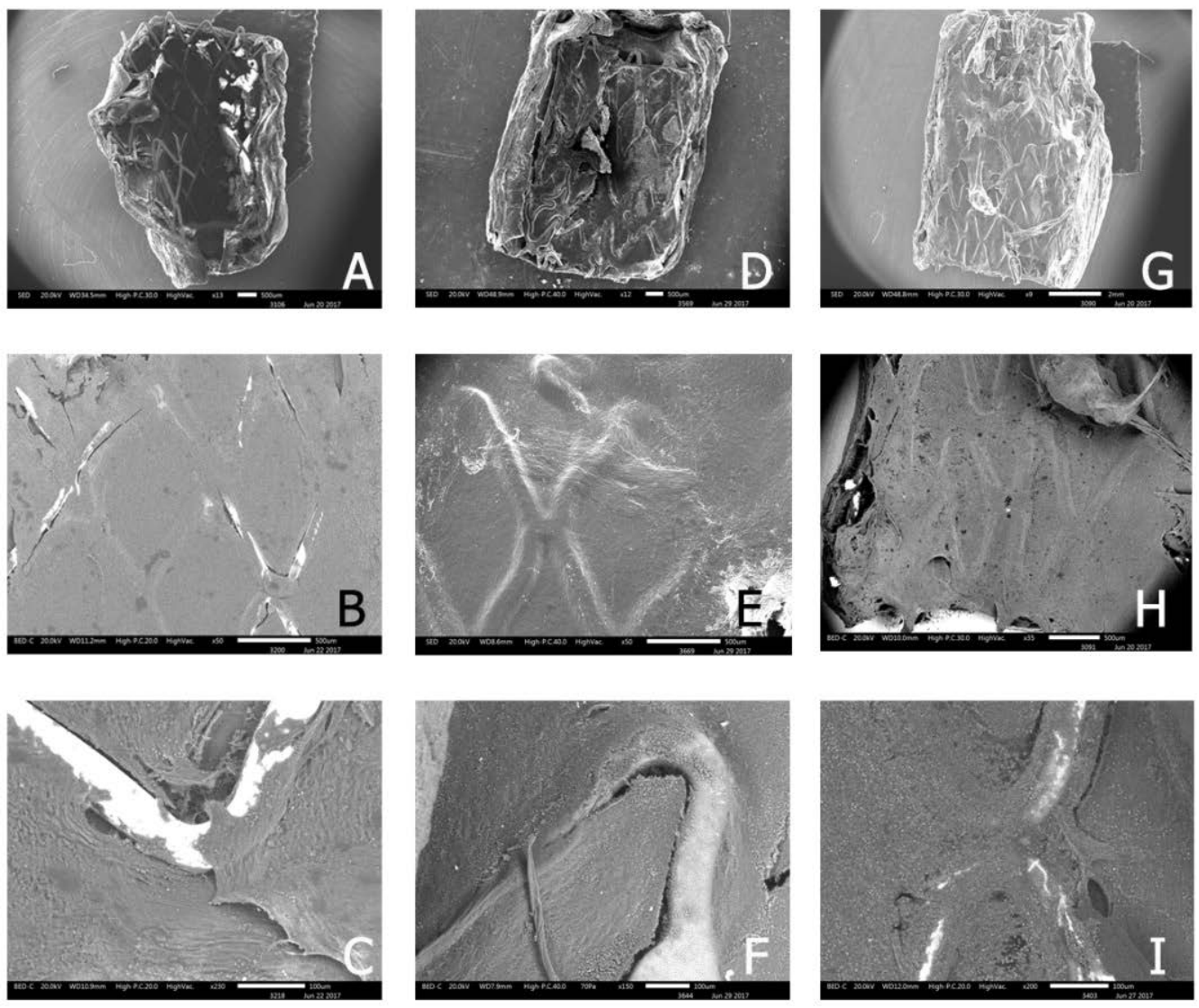

Figure 1: Representative scanning electron microscopy images of 14-days stented rabbit iliac arteries with different magnification. A-C-Stent \#1, D-F-Stent \#2, G-I-Stent \#3 (Control). A,D,G-The common view of segments obtained with SED, average magnification $10 x ; B, E, H$-the view of several crowns of struts obtained with BED, average magnification 40x; C, F, I-the detailed view of struts apex obtained with BED, average magnification 200x. 
At the same time, the coating integrity detailed investigation demonstrated that the Stent \#1 was characterized by the absence of polymer coating on the struts unlike the Stent \#2 and the Control stent those preserved almost intact coating (Figure 2). The samples with the Stent \#2 and Control demonstrated good tissue adhesion to struts whereas Stent \#1 demonstrated lack of tissue adhesion to struts.

\section{Stents evaluation at $\mathbf{2 8}$ days}

Overall, at 28 day, all stents were well covered with endothelium (uncovered area for all stents did not exceed $0.13 \% \pm 0.1 \%, P=0,015)$. In Figure 3, the profile of the stents could be observed, but at the same time the BED-images (Figure 3) confirms the uniformity of tissue. However, the Stent \#2 and Control still had retained uniform polymer coatings (Figure 4). Stent \#1 did not show any evidence of coating presence as noted at 14 day.

\section{Discussion}

Current generation of Drug Eluting Stents (DES) significantly reduce restenosis as compared to BMS. At the same time, a number of clinical studies demonstrate
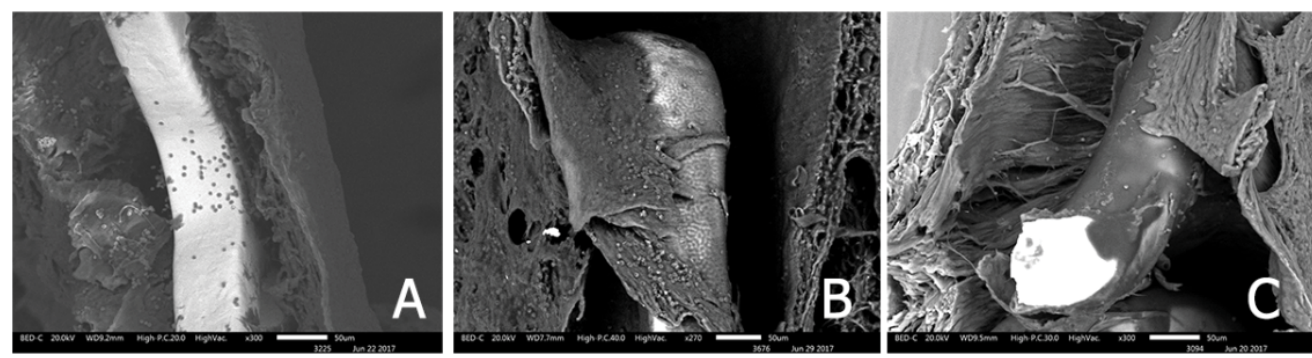

Figure 2: Representative BED-images of samples edges after longitudinal cutting. Samples were harvested after 14 days of implantation. Bright-white areas represent the bare metal surfaces. A-Stent \#1, B-Stent \#2, C-Stent \#3 (Control). Average magnification 300x.
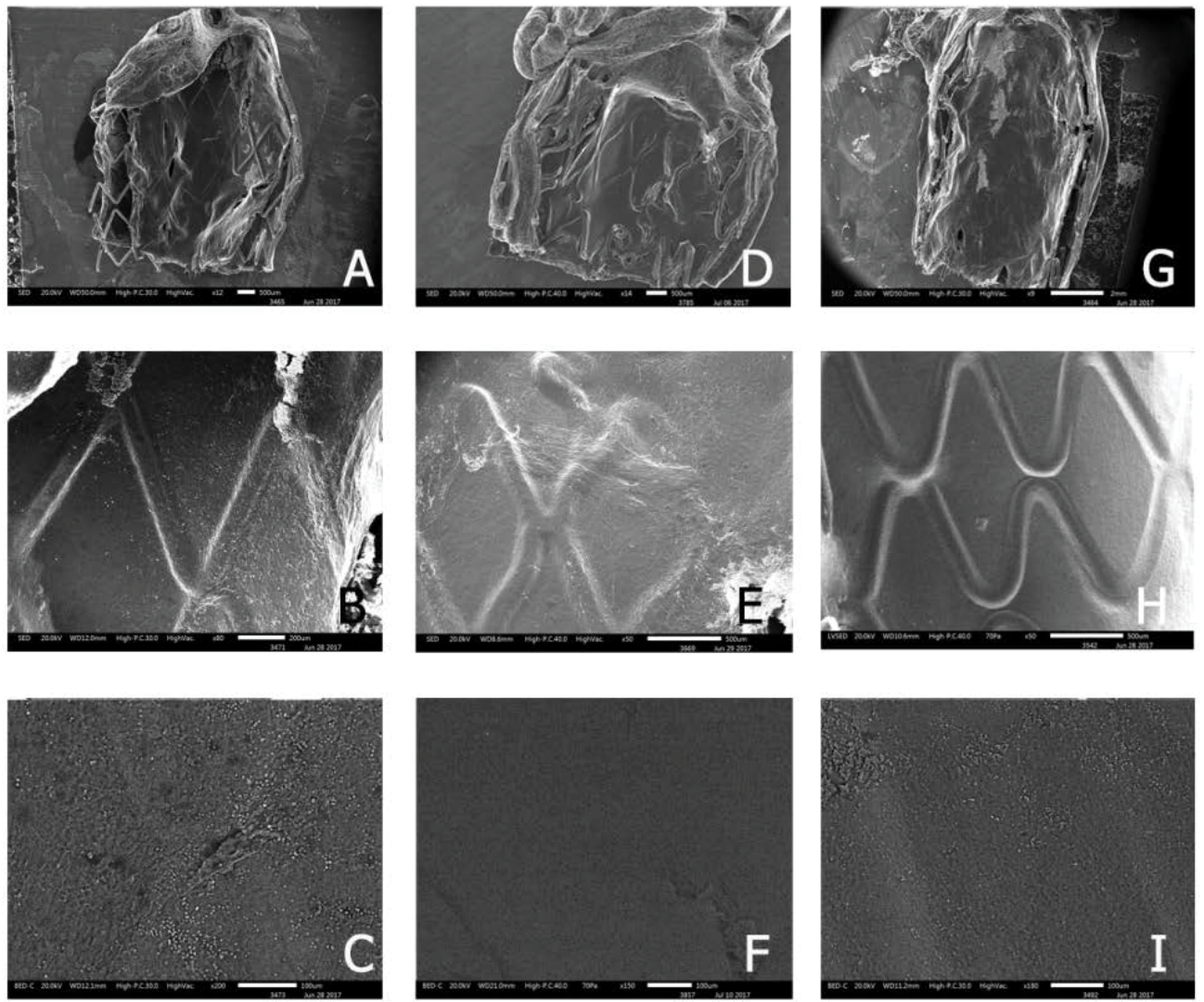

Figure 3: Representative scanning electron microscopy images of 28-days stented rabbit iliac arteries. A-C-Stent \#1, D-F-Stent \#2, G-I-Stent \#3 (Control). A,D,G-SED, average magnification x10; B, E, H-BED, average magnification x50; C, F, I-BED, average magnification 180x. 

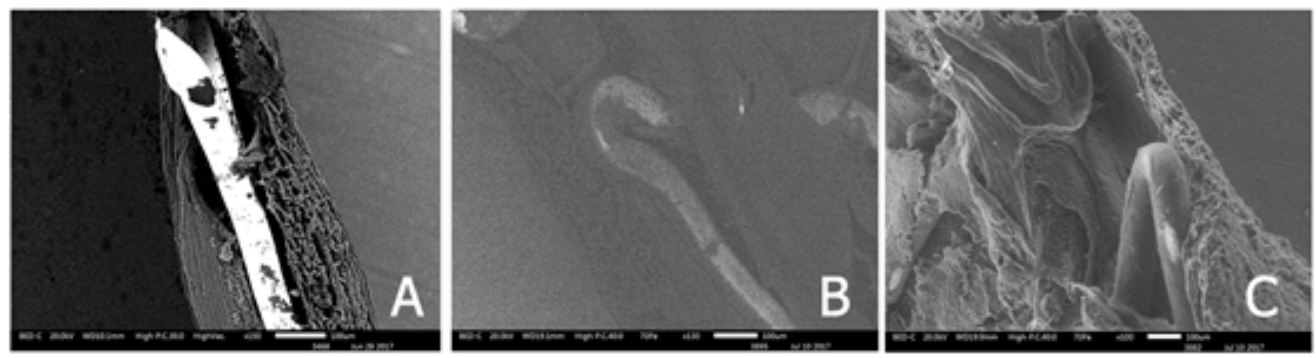

Figure 4: Representative BED-images of iliac arteries edges after longitudinal cutting. Samples were harvested after 28 days of implantation. Bright-white areas represent the bare metal surfaces. A-Stent \#1, B-Stent \#2, C-Stent \#3 (Control). Average magnification 130x.

dependence between strut endothelialization rate and the rate of late stent thrombosis [8]. Recent clinical and non-clinical studies of DES identified several factors that are associated with the stent thrombosis. So, it is very important for DES developers to understand and predict the biological effect of different DES features at the earliest.

Modern stent developers and manufacturers have a limited number of materials for stent coating at their disposal (paclitaxel and members of limus family as drugs, polylactic and polyglycolic acid derivates as biodegradable polymers and a few types of durable polymers: fluoropolymer, SIBS, BioLinx blend etc.). So, in case of similarity of drug-polymer formulation, similar biological effect and clinical results may be expected. It may even be possible to apply clinical data from one DES stent to the others (with same coating formulation).

One of the key factors that affect stent endothelialization is surface strut morphology of various DES platforms. Nevertheless, there is lack of published data regarding influence of various morphological characteristics of polymer coating of DES on biological response of vessel wall and vascular healing.

We compared two biodegradable polymer coated DES having similar material composition (cobalt chromium bare metal base, polylactide-based coating and Sirolimus) with the third one, coated by durable polymer composition with Zotarolimus. In previous bench studies by Kapustin et al. [3] Stent \#1 is characterized by non-uniform coating with a lot of irregularities such as cracks, thinner layer and large bare metal areas. On the contrary, the Stent \#2 [6] and Control [7] in previous bench studies demonstrated smooth uniform polymeric coating without any significant defects. However, mechanical stress during delivery and deployment may lead to coating damage, especially in case of biodegradable PLLA coatings due to polymer structure [9]. Such damages could lead to micro fractures that promote thrombosis or appearance of bare metal areas. Thus, it is beneficial to supplement bench testing with animal studies to better estimate the influence of coating morphology to the in vivo stent performance.

In presented in vivo study, different stents demonstrated different proportion of endothelium covered/uncovered area (Figures 1 and 2). Thus, at 14 day, none of the stent demonstrated the full covering of endothelium, but as it can be observed at Figure $1 \mathrm{~B}$ and $\mathrm{C}$, the Stent \#1 has the highest value of uncovered areas. The following measurements of percent of uncovered struts confirmed this assumption. The lowest value of uncovered area was for Control $(0.9 \% \pm 0.6 \%)$ and the difference between Stent \#1 and Stent \#2 was found significant $(2.75 \% \pm 2.38$ vs. $1.37 \% \pm 1.14 \%$, $\mathrm{P}=0.01)$. The obtained results are consistent with the results published earlier [10] for atherosclerotic rabbit iliac artery model.

The edges of prepared samples of stented vessel were also investigated. In these areas, we can assess stent strut position under newly formed endothelium and evaluate strut surface (coating) state as well. As it could be seen in Figures 3 and 4, even at 14 day Stent \#1 was characterized with the absence of polymer coating on the struts unlike the Stent \#2 and the Control, where both preserved almost intact coating at day 14 and 28 (Figures 3 and 4). Besides, the samples with Stent \#2 and Control demonstrated good tissue adhesion to struts compare to Stent \#1 that was found unattached from the outer and inner sides of vessel (Figure 5).

A recent analogous comprehensive study demonstrated that biodegradable polylactide-based coating is mostly preserved at 28 day and partially preserved at 90 and 180 day in rabbit iliac model [9]. In overall, these findings demonstrate that different initial coating morphology and integrity result in different durability and subsequently biological outcomes, even if the same polymers and drugs are 

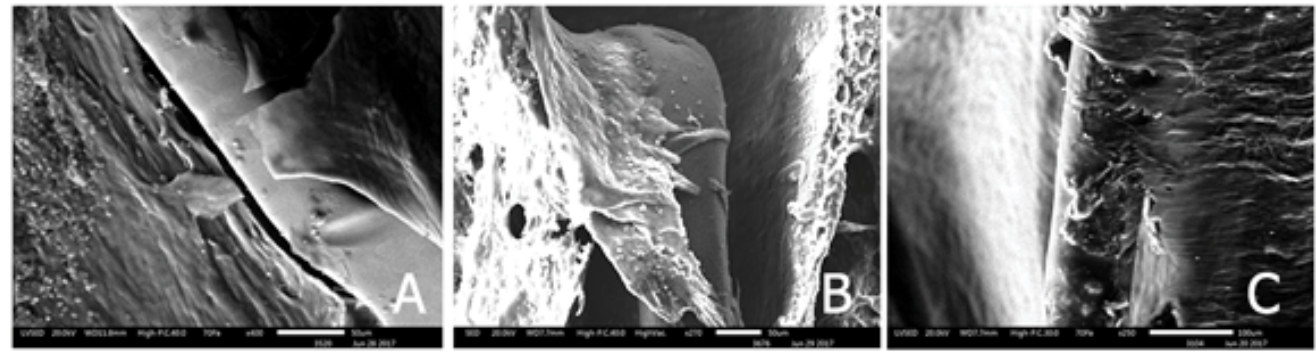

Figure 5: Representative images of stent struts, placed near the cut area after 14 days of implantation. Stent \#2 and Control demonstrated good tissue adhesion in contrast to Stent \#1. A-Stent \#1, magnification $\times 400, B-S t e n t \# 2, C-S t e n t ~ \# 3$ (Control), average magnification 250x.

used. Comprehensive non-clinical and bench top characterizations are therefore necessary to demonstrate each DES platform safety, efficacy and durability prior to any clinical studies.

\section{Conclusion}

We have shown that DES with similar drugpolymer formulation but different coating morphology demonstrates different results after implantation. At the same time, DES with different coating formulation, but similar morphology demonstrates similar biological effect. Thus, our study confirms that not only coating formulation but also coating morphology and topology are critical for stent interaction with vessel wall and endothelialization. These results are aligned with our previous study that demonstrated importance of comprehensive evaluation of coating by different complementary instrumental methods in order to predict (and avoid) potential negative effects early. In clinical practice, excessive irregularities of the polymer coating could increase platelet aggregation on the surface of stent and also impair endothelialization because of poor adhesion of endothelial cells to stent surface.
Actually, due to almost complete loss of coating, in our study Stent \#1 was observed closer to BMS than other DES along with the added issue of potential negative effect of coating stripping off. To confirm these findings further preclinical and clinical research is required.

\section{Future Work}

Future work will be devoted to detailed study of influence of the rate of polymer coating degradation on vascular healing in vivo.

\section{Acknowledgements}

The authors thank to the professor E.V. Suleimanov and Ph.D. A.V. Boryakov (Center of Collective Use "New Materials and Resource-Saving Technologies" of National Research Lobachevsky State University of Nizhni Novgorod) for their valuable technical assistance in scanning electron microscopy research.

\section{Executive summary}

Background: New generation of DES significantly reduce restenosis as compared to BMS. At the same time, a number of clinical studies demonstrate dependence between strut endothelialization rate and rate of late stent thrombosis. Modern stent developers and manufacturers have a limited number of materials for stent coating. Theoretically it could be assumed that similar drug polymer formulation may yield similar biological effect and clinical results and it is even possible to extrapolate clinical data from one stent model to the others (with same coating formulation).

Methods and findings: We evaluated the biological response and endothelization rate of different DES with various drug-polymer formulation after implantation in rabbit iliac artery. Coating surface morphology of each stent was assessed by scanning electron microscopy (SEM) at 14 and 28-day of implantation.

Conclusions: It was shown that DES with similar drug-polymer formulation but different morphology demonstrate the different results after implantation, and at the same time, DES with different coating formulation but similar morphology demonstrate similar biological effect. Thus, our study confirms that not only coating formulation but also coating morphology and topology are critical for stent biocompatibility and endothelization rate. Future work will be devoted to detailed in vivo study of influence of the rate of polymer coating degradation on vascular healing. 


\section{References}

1. Palmerini T, Benedetto U, Biondi-Zoccai G, et al. Long-term safety of drug-eluting and bare-metal stents: evidence from a comprehensive network meta-analysis. J. Am. Coll. Cardiol. 65 (23): 2496-2507 (2015)

2. Basalus MW, Joner M, von Birgelen C, Byrne RA. Polymer coatings on drug-eluting stents: Samson's hair and Achilles' heel? EuroIntervention. 9: 302-305 (2013).

3. Kapustin D, Dmitrieva T, Rashkovskiy A. Complex application of instrumental analytical methods for detection and characteristics of polymer coating defects in drug-eluting stents. Interv. Cardiol. 9(3): 089-105 (2017).

4. vonBirgelen C. Very thin strut biodegradable polymer everolimus-eluting and sirolimus-eluting stents versus durable polymer zotarolimus-eluting stents in allcomers with coronary artery disease (BIO-RESORT): a three-arm, randomised, noninferiority trial. The Lancet. 388 (10060): 2607-2617 (2016).

5. Lee JM, Lee J, Jeong H, et al. Development of a Rabbit Model for a Preclinical Comparison of Coronary Stent Types In-Vivo. Korean. Circ. J. 43: 713-722 (2013).
6. Seth A. Moving Towards Biomimicry-The Development of the Novel BioMime $^{\mathrm{Tm}}$ Sirolimus-eluting Coronary Stent System. Eur. Cardiol. 6(2): 78-82 (2010).

7. Lanzer P. Catheter-Based Cardiovascular Interventions: A Knowledge-Based Approach. Springer: Verlag Berlin Heidelberg. pp: 642 (2013)

8. Reejhsinghani R, Lotfi AS. Prevention of stent thrombosis: challenges and solutions. Vascular Health and Risk Management, 11:93-106 (2015)

9. Devito F. Bioresorbable vascular scaffolds: design, clinical trials, and current applications. Coron. Art. Dis. 27: 151-158 (2016)

10. Nakazawa G, Torii S, Ijichi T, et al. Comparison of Vascular Responses Following New-Generation Biodegradable and Durable Polymer-Based Drug-Eluting Stent Implantation in an Atherosclerotic Rabbit Iliac Artery Model. J. Am. Heart. Assoc. 5(10): 562 (2016)

11. Yazdani S, Sheehy A, Pacetti S, Rittlemeyer B, Kolodgie F, Virmani R. Stent Coating Integrity of Durable and Biodegradable Coated Drug Eluting Stents. J. Interv. Cardiol. 29(5): 483-490 (2016). 